\title{
Parametric Excitation of Alfvén Waves by Gravitational Radiation
}

\author{
M. Servin, G.Brodin and M. Bradley \\ Department of Plasma Physics, Umeå University, SE-901 87 Umeå, Sweden
}

M. Marklund

Department of Electromagnetics, Chalmers University of Technology, SE-412 96 Göteborg, Sweden

\begin{abstract}
We consider the parametric excitation of Alfvén waves by gravitational radiation propagating on a Minkowski background, parallel to an external magnetic field. As a starting point, standard ideal MHD equations incorporating the curvature of space-time has been derived. The growth rate of the Alfvén waves has been calculated, using the normal mode approach. Various astrophysical applications of our investigations are discussed, and finally we demonstrate that the coupling coefficients of the interacting modes fulfill the Manley-Rowe relations.
\end{abstract}

\section{INTRODUCTION}

Interaction of electromagnetic fields with gravitational radiation has been studied by several authors [1-19]. Besides a purely theoretical interest in such phenomena, there is a number of different applications. For example in astrophysics [1-5], in cosmology [6] and under laboratory conditions [7-10], where - in the later case - the goal is to find suitable mechanisms to detect gravitational radiation. Furthermore, there are many examples of gravitational wave interaction that may take place in plasmas. This has been studied during the eighties by a group at the Kazan School of gravitation (see for example $[1,10,11]$ and references therein) and more recently by Refs. 4, 12, 13 and 14.

In Ref. 12 it was shown that parametric excitation of high frequency plasma waves by gravitational radiation may take place. Due to the frequency matching conditions, however, the plasma must be very thin for that process to be possible, and the amount of energy transfer is therefore limited. In the present paper we will thus consider parametric excitation of low frequency MHD waves by gravitational waves, which - in contrast - may take place in a comparatively dense plasma. The relevance of this problem for the conversion of gravitational wave energy to the plasma inside supernovas has previously been discussed by Ref. 14. However, due to the complexity of the physical situation, a highly idealized model will be studied, where a one dimensional monochromatic gravitational wave - superimposed on a flat background metric - propagates through a homogeneous two component plasma.

The organization of the paper is as follows: In section II idealized MHD-equations incorporating the effects of the gravitational wave are derived, starting from covariant two-fluid equations. In section III parametric excitation of shear Alfvén and magnetosonic waves are considered, the three wave coupling coefficients are derived and the growth rate is found. By adding a phenomenological resistivity to the equations, the threshold value of the gravitational amplitude is also calculated. Finally, in section IV, our results are summarized and theoretical considerations like energy conservation properties and the fulfillment of Manley-Rowe relations as well as possible applications are discussed.

\section{RELATIVISTIC MHD-EQUATIONS}

In order to obtain general relativistic fluid equations governing a plasma we begin by considering a system consisting of a charged perfect fluid and an electromagnetic field [20]. Introducing the restframe scalar quantities: mass density (or rather energy density times $\left.1 / c^{2}\right) \rho_{(m)}$, charge density $\rho_{(q)}$, pressure $p$ and the 4 -velocity field (or fluid velocity) $u^{\mu} \equiv d x^{\mu} / d \tau$ and 4-current density $j^{\mu} \equiv \rho_{(q)} u^{\mu}$, where $\tau$ is the proper time and $x^{\mu}$ coordinates in the lab frame, this system is characterized by having the energy-momentum tensor $T^{\mu \nu}=T_{(f l)}^{\mu \nu}+T_{(e m)}^{\mu \nu}$, where

$$
\begin{aligned}
T_{(f l)}^{\mu \nu} & \equiv\left(\rho_{(m)}+\frac{p}{c^{2}}\right) u^{\mu} u^{\nu}-p g^{\mu \nu} \\
T_{(e m)}^{\mu \nu} & \equiv \frac{1}{\mu_{0}}\left(F^{\mu \tau} F_{\tau}{ }^{\nu}-\frac{1}{4} g^{\mu \nu} F^{\tau \sigma} F_{\sigma \tau}\right)
\end{aligned}
$$

and $F_{\mu \nu}$ is the electromagnetic field tensor satisfying Maxwell's equations

$$
\begin{gathered}
F_{; \nu}^{\mu \nu}=-\mu_{0} j^{\mu} \\
F_{\mu \nu ; \sigma}+F_{\nu \sigma ; \mu}+F_{\sigma \mu ; \nu}=0
\end{gathered}
$$

We have adopted the convention that Greek suffixes $\mu, \nu, .$. have the range $0,1,2,3$ and $i, j, .$. have the range $1,2,3$ and the metric tensor $g^{\mu \nu}$ has the signature (+ - - ).

The conservation laws of the system follows from that the 4-divergence of the energy-momentum tensor vanishes, i.e. $T_{; \mu}^{\mu \nu}=0$, and with the use of Maxwell's equations one gets 


$$
\begin{gathered}
\left(\rho_{(m)} u^{\mu}\right)_{; \mu}+\frac{p}{c^{2}} u_{; \mu}^{\mu}=0 \\
\left(\rho_{(m)}+\frac{p}{c^{2}}\right) u^{\mu} u_{; \mu}^{\nu}=\left(g^{\mu \nu}-\frac{1}{c^{2}} u^{\mu} u^{\nu}\right) p_{, \mu}+F_{\mu}^{\nu} j^{\mu}
\end{gathered}
$$

where eq.(3) is obtained by projection along the 4velocity $u^{\mu}$. This equation is identified as energy balance in the rest-frame of matter and gives the equation of continuity (mass conservation) in the non relativistic limit. Equation (位) gives for $\nu=0$ the energy balance (modulo the content of (3), i.e., energy balance in the non relativistic limit) and for $\nu=1,2,3$ momentum balance.

Under the conditions of low internal energy the fluid description of a plasma can be simplified by putting, for each particle species of the plasma, $\rho_{(m)}=m n$ where $n$ is the restframe particle density and $m$ is the particle mass. Also without these restrictions we may put $\rho_{(q)}=q n$ where $q$ is the particle charge. Suppose we have a plasma consisting of two species of particles oppositely charged (i.e. $q_{1} / q_{2}=-1$ ), but, in general, with distinct masses. For each species we assign a fluid satisfying the equations (3) and (44). The fluids are assumed interpenetrating and interacting through the electromagnetic field and, in general, the gravitational field. We neglect the effect of particle collisions. If we assume non-relativistic pressure, i.e. such that $m n \gg p / c^{2}$, and non-relativistic fluid velocities - in the sense that we may neglect quadratic terms in $1 / c$ in the $\nu \neq 0$ components of eq.(化) - then we have, for each of the two fluids, equations for conservation of particles (or mass) and momentum in the form

$$
\begin{aligned}
\left(n u^{\mu}\right)_{; \mu} & =0 \\
m n u^{\mu} u_{; \mu}^{i} & =g^{i j} p_{, j}+F_{\mu}^{i} q n u^{\mu}
\end{aligned}
$$

Maxwell's equations remain the same if we let $j_{\mu}$ be the total current density.

Under the conditions that for both species $\partial_{t} \sim \omega \ll \omega_{c}$ and $C_{A}^{2} \ll c^{2}$, this two-fluid description can be cast into a set of single-fluid equations. By $\partial_{t} \sim \omega$ we mean that a characteristic frequency, $\omega$, can be assigned to the time variations in the dynamical quantities [21]. We use $\omega_{c} \equiv|q| B / m$ for the cyclotron frequency, $B$ is the magnetic field intensity (which can be obtained from the Lorentz frame components of the electromagnetic field tensor) and $C_{A}$ is the Alfvén velocity, defined by $C_{A}^{2}=B^{2} / \mu_{0} m n$. Under the above conditions it follows that the two fluid velocities are approximately equal and that the particle densities may be regarded as exactly equal. Furthermore, we let the equation of state be the one of isothermal compression and assume $\left|u^{\mu} u_{; \mu}^{i}\right| \ll\left|F_{0}^{i} q n u^{0}\right| / m n \propto\left|F_{j}^{i} q n u^{j}\right| / m n$ (meaning that the electric and magnetic forces approximately balance each other). In light of this, we obtain the following set of single fluid equations (MHD-equations)

$$
\left(n u^{\mu}\right)_{; \mu}=0
$$

$$
\begin{gathered}
\left(m_{(1)}+m_{(2)}\right) n u^{\mu} u_{; \mu}^{i}=g^{i j} p_{, j}+F_{\mu}^{i} j^{\mu} \\
F_{\mu}^{i} u^{\mu}=-\frac{1}{n q_{(1)}} g^{i j}\left(\frac{m_{(2)} p_{(1), j}-m_{(1)} p_{(2), j}}{m_{(1)}+m_{(2)}}\right) \\
p_{, i}=k_{B} T n_{, i}
\end{gathered}
$$

Equation (5) and (6) are obtained by adding the two particle conservation equations and the two equations of momentum balance, respectively, and setting the velocities equal (when added), letting $j$ be the total current density, $p$ the total pressure and $T \equiv T_{(1)}+T_{(2)}$. The suffixes (1) and (2) refers to the two distinct particle species. By subtracting the two equations of momentum conservation, where the terms $u^{\mu} u_{; \mu}^{i}$ have been neglected, one finds eq. (7), which we refer to as the generalized Ohm's law.

Note that if the right hand side of eq. (7) is negligible as compared to $F_{0}^{\imath} u^{0}$ and $F_{j}^{\imath} u^{j}$ this equation simply reads $F_{\mu}^{i} u^{\mu}=0$ and we can then refer to the singlefluid equations as the ideal MHD-equations. In the following we limit ourselves to this case. Note that the MHD-equations are not independent of Maxwell's and Einstein's field equations, since we used Maxwell's equations and $T_{; \mu}^{\mu \nu}=0$, that follows from Einstein's equations, in deriving them.

We now consider gravitational radiation on a Minkowski background treating the plasma as a testfluid. Thus, the plasma back scattering effect on the gravitational field is lost. The gravitational radiation is chosen to be weak gravitational waves in the transverse traceless (TT) gauge propagating in the $x^{3}$-direction. This plane wave solution of the linearized Einstein field equations can be written

$$
\begin{aligned}
d s^{2}= & c^{2} d t^{2}-\left(1-h_{+}\right) d x^{2}-\left(1+h_{+}\right) d y^{2} \\
& +2 h_{\times} d x d y-d z^{2}
\end{aligned}
$$

where $h \equiv \tilde{h} e^{i k_{\mu} x^{\mu}}+$ c.c. and $|\tilde{h}| \ll 1$ with the wave vector $\left[k^{\mu}\right]=(\omega / c, 0,0, k)$ satisfying the dispersion relation $k^{\mu} k_{\mu}=0$. In all the following calculations we neglect terms that are quadratic in $\tilde{h}$ or higher. The metric tensor can thus be written as $g_{\mu \nu}=\eta_{\mu \nu}+h_{\mu \nu}$ where $\eta_{\mu \nu}$ is the metric tensor of Minkowski space and $h_{\mu \nu}$ represents the small, $\left|h_{\mu \nu}\right| \ll 1$, fluctuation in the gravitational field.

The nonzero Christoffel symbols are then calculated to

$$
\begin{gathered}
\Gamma_{01}^{1}=-\Gamma_{02}^{2}=\Gamma_{11}^{0}=-\Gamma_{22}^{0}=-\Gamma_{13}^{1} \\
=\Gamma_{23}^{2}=\Gamma_{11}^{3}=-\Gamma_{22}^{3}=\frac{1}{2} \dot{h}_{+} \\
\Gamma_{02}^{1}=\Gamma_{01}^{2}=-\Gamma_{23}^{1}=-\Gamma_{13}^{2}=\Gamma_{12}^{3}=\Gamma_{12}^{0}=\frac{1}{2} \dot{h}_{\times}
\end{gathered}
$$


where $\dot{h} \equiv \partial h / \partial \xi$ and $\xi \equiv x^{3}-x^{0}$.

By expanding the covariant derivative, eq.(司) becomes $\left(n u^{\mu}\right)_{, \mu}=0$ as a result of $\Gamma_{\nu \mu}^{\mu} u^{\nu}=0$ and - noting that $g^{\mu \nu}=\eta^{\mu \nu}-h^{\mu \nu}$ to first order, such that $g^{i j}=-\delta^{i j}-h^{i j}$ - eq.(6) reads

$$
m n u^{\mu} u_{, \mu}^{i}+G^{i}=-\delta^{i j} p_{, j}-h^{i j} p_{, j}+F^{i}{ }_{\mu} j^{\mu}
$$

where we have introduced $G^{i}=m n \Gamma_{\nu \mu}^{i} u^{\nu} u^{\mu}$ and $m=$ $m_{(1)}+m_{(2)}$.

Next, we perform the same expansion in Maxwell's equations and rewrite them in terms of the electromagnetic field tensor in the form $F^{\mu}{ }_{\nu}$. The idea is to express all field tensor terms in the same form, preferably the one that gives the most simple expressions. We can separate $F_{; \nu}^{\mu \nu}=-\mu_{0} j^{\mu}$ into two equations. Setting $\mu=0$ we obtain a Poisson-like equation which we discard - since in the MHD-regime $j^{0} \approx 0$. Setting $\mu=i$ we read off "Ampere's law",

$$
\delta^{j k} F_{k, j}^{i}=\mu_{0} j^{i}-\left(h^{j \nu} F^{i}{ }_{\nu}\right)_{, j}+\Gamma^{i}{ }_{\tau j} g^{j \nu} F^{\tau}{ }_{\nu}+\Gamma^{j}{ }_{\tau j} g^{\tau \nu} F^{i}{ }_{\nu}
$$

From eq.(2), which by symmetry in $F^{\mu \nu}$ is equivalent to $F_{\mu \nu, \sigma}+F_{\nu \sigma, \mu}+F_{\sigma \mu, \nu}=0$, we obtain a number of trivial identities, a generalized equation for $\nabla \cdot \mathbf{B}$ and "Faraday's law":

$$
\begin{aligned}
F_{0,2}^{3}-F_{2,3}^{0}+F_{3,0}^{2}= & \left(h_{\times} F_{3}^{1}-h_{+} F_{3}^{2}\right)_{, 0} \\
-F_{0,1}^{3}+F_{1,3}^{0}-F_{3,0}^{1}= & -\left(h_{+} F_{3}^{1}+h_{\times} F_{3}^{2}\right)_{, 0} \\
F_{0,1}^{2}-F_{1,2}^{0}+F_{2,0}^{1}= & \left(h_{+} F_{2}^{1}+h_{\times} F_{2}^{2}\right)_{, 0} \\
& +\left(h_{\times} F_{0}^{1}-h_{+} F_{0}^{2}\right)_{, 1}
\end{aligned}
$$

For notational purposes it is convenient to introduce an abstract basis $\{\hat{\mathbf{x}}, \hat{\mathbf{y}}, \hat{\mathbf{z}}\}$. The one-fluid equations and the Maxwell's equations above can then be written in a vector representation with an algebraic structure identical to the Euclidean. We define

$$
\begin{aligned}
\mathbf{x} & \equiv x \hat{\mathbf{x}}+y \hat{\mathbf{y}}+z \hat{\mathbf{z}} \equiv x^{1} \hat{\mathbf{x}}+x^{2} \hat{\mathbf{y}}+x^{3} \hat{\mathbf{z}} \\
\mathbf{v} & \equiv v_{x} \hat{\mathbf{x}}+v_{y} \hat{\mathbf{y}}+v_{z} \hat{\mathbf{z}} \equiv u^{1} \hat{\mathbf{x}}+u^{2} \hat{\mathbf{y}}+u^{3} \hat{\mathbf{z}} \\
\mathbf{j} & \equiv j_{x} \hat{\mathbf{x}}+j_{y} \hat{\mathbf{y}}+j_{z} \hat{\mathbf{z}} \equiv j^{1} \hat{\mathbf{x}}+j^{2} \hat{\mathbf{y}}+j^{3} \hat{\mathbf{z}} \\
\mathbf{E} & \equiv E_{x} \hat{\mathbf{x}}+E_{y} \hat{\mathbf{y}}+E_{z} \hat{\mathbf{z}} \equiv c F_{1}^{0} \hat{\mathbf{x}}+c F^{0} \hat{\mathbf{y}}+c F^{0}{ }_{3} \hat{\mathbf{z}} \\
\mathbf{B} & \equiv B_{x} \hat{\mathbf{x}}+B_{y} \hat{\mathbf{y}}+B_{z} \hat{\mathbf{z}} \equiv F_{3}^{2} \hat{\mathbf{x}}+F_{1}^{3} \hat{\mathbf{y}}+F_{{ }_{2}}^{1} \hat{\mathbf{z}} \\
\nabla & \equiv \partial_{x} \hat{\mathbf{x}}+\partial_{y} \hat{\mathbf{y}}+\partial_{z} \hat{\mathbf{z}} \equiv \frac{\partial}{\partial x^{1}} \hat{\mathbf{x}}+\frac{\partial}{\partial x^{2}} \hat{\mathbf{y}}+\frac{\partial}{\partial x^{3}} \hat{\mathbf{z}}
\end{aligned}
$$

(Note that these quantities differ to first order in $\tilde{h}$ from what an observer in the lab system would measure.) One then obtains the following set of equations governing the plasma

$$
\begin{aligned}
m n\left(\partial_{t} \mathbf{v}+(\mathbf{v} \cdot \nabla) \mathbf{v}\right) & =\mathbf{j} \times \mathbf{B}-\nabla p+\mathbf{g} \\
\mathbf{E}+\mathbf{v} \times \mathbf{B} & =0
\end{aligned}
$$

$$
\begin{aligned}
\partial_{t} n+\nabla \cdot(n \mathbf{v}) & =0 \\
\nabla p & =k_{B} T \nabla n \\
\nabla \times \mathbf{B} & =\mu_{0} \mathbf{j}+\mathbf{j}_{E} \\
\partial_{t} \mathbf{B}+\nabla \times \mathbf{E} & =-\mathbf{j}_{B}
\end{aligned}
$$

where

$$
\begin{aligned}
\mathbf{g}= & -\mathbf{G}+\mathbf{g}_{\text {pressure }}+\mathbf{g}_{\mathrm{em}-\text { coupling }} \\
\mathbf{G}=- & m n\left[\left(v_{z}-c\right)\left(\dot{h}_{+} v_{x}+\dot{h}_{\times} v_{y}\right),\right. \\
& \left(v_{z}-c\right)\left(\dot{h}_{\times} v_{x}-\dot{h}_{+} v_{y}\right), \\
& \left.\frac{1}{2} \dot{h}_{+}\left(v_{x}^{2}-v_{y}^{2}\right)-\dot{h}_{\times} v_{x} v_{y}\right] \\
\mathbf{g}_{\text {pressure }}=- & {\left[h_{+} \partial_{x} p+h_{\times} \partial_{y} p, h_{\times} \partial_{x} p-h_{+} \partial_{y} p, 0\right] } \\
\mathbf{g}_{\text {em-coupling }}= & {\left[-h_{\times} B_{z} j_{x}-\left(h_{+} B_{y}-h_{\times} B_{x}\right) j_{z},\right.} \\
& \left.2 h_{+} B_{z} j_{x}+h_{\times} B_{z} j_{y},-\left(h_{+} B_{x}+h_{\times} B_{y}\right) j_{y}\right] \\
\mathbf{j}_{E} \equiv & {\left[\partial_{y}\left(h_{+} B_{z}\right)+\partial_{z}\left(h_{+} B_{y}-h_{\times} B_{x}\right),\right.} \\
& \left.-\partial_{x}\left(h_{+} B_{z}\right),-\partial_{x}\left(h_{+} B_{y}-h_{\times} B_{y}\right)\right] \\
\mathbf{j}_{B} \equiv & {\left[\partial_{t}\left(h_{+} B_{x}+h_{\times} B_{y}\right), 0,-\partial_{t}\left(h_{+} B_{z}\right)\right] }
\end{aligned}
$$

We want to point out that terms of the order $E \dot{h}_{\times} / c$ in $\mathbf{j}_{E}$ have been neglected, since $E / B$ is of the order $C_{A}$ in ideal MHD theory. Furthermore, note that $c F_{0}^{1} \neq$ $c F_{1}^{0} \equiv E_{x}$, etc., which is the origin of some of the terms appearing in the expressions for $\mathbf{g}_{\mathrm{em}-\text {-coupling }}, \mathbf{j}_{E}$ and $\mathbf{j}_{B}$. In addition to eqs.(11)-(16) Maxwell's equations produce constraints (e.g. for $\nabla \cdot \mathbf{B}$ ), however it is easy to verify that these constraints are propagated by the equations of time evolution, (11), (13) and (16).

\section{WAVE-WAVE INTERACTIONS}

From now on we will consider resonant three-wave interaction between gravitational radiation and MHDwaves. Such processes may occur whenever quadratic nonlinear terms, such as the right hand sides of eqs. (11)(16), are present, and the dispersion relations of the interacting waves allow for the frequency and wave-vector matching conditions to be fulfilled. For a general review of resonant three wave interaction in plasmas, see e.g. Ref. 22.

In the absence of any waves we assume to have the configuration of a static homogeneous, $n=n^{(0)}$, magnetized, $\mathbf{B}=\mathbf{B}^{(0)}$, plasma in Minkowski space. Cartesian coordinates are chosen $\left(x^{\mu}=(c t, x, y, z)\right)$ for a frame in which the velocity field (and the current density field) vanishes. The gravitational waves are then inferred as small perturbations to the Minkowski background, as in the previous section, and the MHD-waves as the existence of the small fluctuations: $n^{(1)}, \mathbf{v}^{(1)}, \mathbf{j}^{(1)}, \mathbf{E}^{(1)}, \mathbf{B}^{(1)}$. Furthermore, in order to simplify the algebra, we make the assumptions that the direction of $\mathbf{B}^{(0)}$ is everywhere parallel to the direction of propagation of the gravitational 
waves, i.e. $\mathbf{B}^{(0)}=B^{(0)} \hat{\mathbf{z}}$, and that the gravitational radiation is polarized such that $h_{+}=0$.

\section{A. Linear Calculations}

It is instructive to first investigate the linearized theory in some detail. Linearizing the equations (11)-(16) in the variables $h_{\times}, n^{(1)}, \mathbf{v}^{(1)}, \mathbf{j}^{(1)}, \mathbf{E}^{(1)}, \mathbf{B}^{(1)}$ we find that the gravitational waves do not drive plasma perturbations linearly. This is a consequence of the direction of propagation of the gravitational wave (parallel to the magnetic field) that was chosen. Similarly the linear plasma perturbations are the ordinary MHD-modes. Fourier analyzing we obtain the dispersion relations for the shear Alfvén wave

$$
D_{A} \equiv \omega^{2}-C_{A}^{2} k_{z}^{2}=0
$$

and for the fast and slow magnetosonic wave

$$
D_{m} \equiv \omega^{4}-\omega^{2} k^{2}\left(C_{S}^{2}+C_{A}^{2}\right)+k_{z}^{2} k^{2} C_{S}^{2} C_{A}^{2}=0 .
$$

The constants introduced are the Alfvén velocity $C_{A}^{2} \equiv$ $B^{(0)^{2}} / m n^{(0)} \mu_{0}$, the thermal velocity $C_{S}^{2} \equiv k_{B} T / m$, and we have used the notation $\mathbf{k}=k_{x} \hat{\mathbf{x}}+k_{z} \hat{\mathbf{z}}$ together with $k=|\mathbf{k}|$. In the next subsection we will consider superposition of MHD-waves, and by expressing all variables in terms of the fluid velocity we can represent the solution as a sum of eigenvectors

$$
\left(\begin{array}{c}
n^{(1)} \\
\mathbf{v}^{(1)} \\
\mathbf{j}^{(1)} \\
\mathbf{E}^{(1)} \\
\mathbf{B}^{(1)}
\end{array}\right)=\sum_{\alpha}\left(\begin{array}{c}
\theta_{\alpha} n^{(0)} \\
\mathbf{v}_{\alpha}^{(1)} \\
\frac{i \theta_{\alpha}}{\mu_{0}} \mathbf{k}_{\alpha} \times \mathbf{B}^{(0)}-\frac{i \sigma_{\alpha}}{\mu_{0}} \mathbf{k}_{\alpha} \times \mathbf{v}_{\alpha}^{(1)} \\
\mathbf{B}^{(0)} \times \mathbf{v}_{\alpha}^{(1)} \\
\theta_{\alpha} \mathbf{B}^{(0)}-\sigma_{\alpha} \mathbf{v}_{\alpha}^{(1)}
\end{array}\right)
$$

where $\theta_{\alpha} \equiv \mathbf{k}_{\alpha} \cdot \mathbf{v}_{\alpha}^{(1)} / \omega_{\alpha}, \sigma_{\alpha} \equiv k_{\alpha z} B^{(0)} / \omega_{\alpha}$ and $\alpha$ is a wave-mode index.

As we intend to study nonlinear wave coupling it is convenient to adopt the normal mode method of approach [22], which typically simplifies the algebra in the nonlinear stage of the calculations. We define a normal mode as a linear combination, $a_{\alpha}$, of the dynamical quantities that to linear order satisfies

$$
\partial_{t} a_{\alpha}+i \omega_{\alpha} a_{\alpha}=0
$$

The dynamical quantities are now only assumed to have harmonic spatial dependence, i.e. $\nabla=i \mathbf{k}_{\alpha}$. From eq. (20) the proper linear combinations are

$$
a_{A}=v_{y}^{(1)}-\frac{\omega_{A}}{k_{A z} B^{(0)}} B_{y}^{(1)}
$$

for the Alfvén mode, and

$$
\begin{aligned}
a_{m}= & n^{(1)}+\chi v_{x}^{(1)}+\frac{n^{(0)} k_{m z}}{\omega_{m}} v_{z}^{(1)} \\
& -\frac{k_{m_{z}} C_{A}^{2} \chi}{B^{(0)} \omega_{m}} B_{x}^{(1)}+\frac{k_{m_{x}} C_{A}^{2} \chi}{B^{(0)} \omega_{m}} B_{z}^{(1)}
\end{aligned}
$$

for the magnetosonic modes, with the frequency-wave number pairs $\left(\omega_{A}, \mathbf{k}_{A}\right)$ and $\left(\omega_{m}, \mathbf{k}_{m}\right)$ satisfying the dispersion relations $D_{A}=0$ and $D_{m}=0$, respectively. The constant $\chi$ is defined as $\chi \equiv n_{0}\left(\omega_{m}^{2}-C_{S}^{2} k_{m_{z}}^{2}\right) / C_{S}^{2} k_{m x} \omega_{m}$. With aid of the corresponding eigenvectors and the relation $v_{z}^{(1)}=v_{x}^{(1)} C_{S}^{2} k_{m x} k_{m z} /\left(\omega_{m}^{2}-C_{S}^{2} k_{m x}^{2}\right)$ we can, after some algebraic manipulations, write the normal modes as

$$
\begin{aligned}
& a_{A}=2 v_{y}^{(1)}=\frac{1}{\omega_{A}} \frac{\partial D_{A}}{\partial \omega_{A}} v_{y}^{(1)} \\
& a_{m}=\frac{n^{(0)}}{\omega_{m}^{2} C_{S}^{2} k_{m x}^{2}} \frac{\partial D_{m}}{\partial \omega_{m}} v_{x}^{(1)} .
\end{aligned}
$$

For the nonlinear calculation we need the eigenvectors expressed in terms of the normal modes, and the final linear results are

$$
\left(\begin{array}{l}
n^{(1)} \\
\mathbf{v}^{(1)} \\
\mathbf{j}^{(1)} \\
\mathbf{E}^{(1)} \\
\mathbf{B}^{(1)}
\end{array}\right)_{A}=a_{A}\left(\begin{array}{c}
0 \\
\left(0, \frac{1}{2}, 0\right) \\
\left(\frac{i B^{(0)} k_{A z}^{2}}{2 \omega_{A} \mu_{0}}, 0,-\frac{i B^{(0)} k_{A x} k_{A z}}{2 \omega_{A} \mu_{0}}\right) \\
\left(-\frac{B^{(0)}}{2}, 0,0\right) \\
\left(0,-\frac{B^{(0)} k_{A z}}{2 \omega_{A}}, 0\right)
\end{array}\right)
$$

and

$$
\left(\begin{array}{l}
n^{(1)} \\
\mathbf{v}^{(1)} \\
\mathbf{j}^{(1)} \\
\mathbf{E}^{(1)} \\
\mathbf{B}^{(1)}
\end{array}\right)_{m}=a_{m} c_{m}\left(\begin{array}{c}
1+\frac{C_{S}^{2} k_{m z}^{2}}{\omega_{m}^{2}-C_{S}^{2} k_{m z}^{2}} \\
\left(\zeta, 0, \zeta \frac{C_{S}^{2} k_{m x} k_{m z}}{\omega_{m}^{2}-C_{2}^{2} k_{m z}^{2}}\right) \\
\left(0,-\frac{i B^{(0)} k_{m}^{2}}{n^{(0)} k_{m x} \mu_{0}}, 0\right) \\
\left(0, \frac{\omega_{m} B^{(0)}}{n^{(0)} k_{m x}}, 0\right) \\
\left(-\frac{k_{m z} B^{(0)}}{n^{(0)} k_{m x}}, 0, \frac{B^{(0)}}{n^{(0)}}\right)
\end{array}\right)
$$

where $c_{m} \equiv \omega_{m}^{2} C_{S}^{2} k_{m x}^{2} / 2\left(\omega_{m}^{4}-k_{m}^{2} k_{m z}^{2} C_{A}^{2} C_{S}^{2}\right)$ and $\zeta \equiv$ $\omega_{m} / n^{(0)} k_{m x}$

\section{B. Nonlinear Calculations}

The aim of this section is to investigate the lowest order nonlinear influence of the gravitational radiation on the MHD modes described above. In particular we are interested in the threshold value (for parametric excitation) of the gravitational amplitude, and the growth rates of the excited MHD waves. We will again assume that the wave vectors lies in $x z$-plane, i.e. $\mathbf{k}=k_{x} \hat{\mathbf{x}}+k_{z} \hat{\mathbf{z}}$ for the MHD-waves, but in contrast to the case of linear wave 
modes this is a restriction made in order to simplify the algebra [23].

We consider coherent three-wave interactions, the three waves being one gravitational wave and two MHDwaves, with the matching conditions

$$
\begin{aligned}
& \omega_{g}=\omega_{I}+\omega_{I I} \\
& \mathbf{k}_{g}=\mathbf{k}_{I}+\mathbf{k}_{I I}
\end{aligned}
$$

where $I$ and $I I$ are indexing the MHD-waves. In the nonlinear regime the normal modes does no longer satisfy eq. (20), but rather

$$
\partial_{t} a_{\alpha}+i \omega_{\alpha} a_{\alpha}=\left(\left[\partial_{t} a_{\alpha}\right]_{\mathrm{n} .1 .}\right)_{\mathbf{k}_{\alpha}}
$$

where n.l. denotes (first order) nonlinear terms and the suffix $\mathbf{k}_{\alpha}$ indicates that terms not oscillating as $e^{i \mathbf{k} \cdot \mathbf{x}}$ vanishes due to rapid oscillations. Explicit forms for the right hand side is found by using the original expressions for the normal modes - eqs. (21) or (22) - together with eqs. (11)-(16).

We let index $I$ denote the magnetosonic wave perturbation, index $I I$ the Alfvén wave perturbation and we use a complex representation (i.e. letting $f \rightarrow f+f^{*}$ for all variables, where the star denotes complex conjugate). Making use of the linear eigenvectors (25) and (26) as approximations in the nonlinear right hand sides, we obtain the coupled mode equations

$$
\begin{aligned}
\partial_{t} a_{I}+i \omega_{I} a_{I} & =C_{I} a_{I I}^{*} h_{\times} \\
\partial_{t} a_{I I}+i \omega_{I I} a_{I I} & =C_{I I} a_{I}^{*} h_{\times}
\end{aligned}
$$

after lengthy but straightforward algebra, where the coupling coefficients are [24]

$$
\begin{aligned}
C_{I} & =-\frac{i}{2} \frac{n_{0} k_{x I}}{\omega_{I}^{2}-C_{A}^{2} k_{I}^{2}} \omega_{I} \omega_{g} \\
C_{I I} & =-\frac{i}{2} \frac{1}{n_{0}} \frac{\omega_{I}^{2} C_{S}^{2} k_{x I}}{\left(\omega_{I}^{4}-C_{S}^{2} C_{A}^{2} k_{I}^{2} k_{z I}^{2}\right)} \omega_{I I} \omega_{g}
\end{aligned}
$$

In deriving the expressions for $C_{I}$ and $C_{I I}$ we have also applied $\mathbf{k}_{I} \approx-\mathbf{k}_{I I}$, which follows from the matching condition (28) together with $C_{A} \ll c$ and the dispersion relations.

From eq. (31) and (32) one may get the incorrect impression that the coupling strength diverges in the limit $C_{S}^{2} \rightarrow 0$. Thus in order to shed some light on our formulas in the cold limit, we first renormalize

$$
\begin{aligned}
& a_{I} \rightarrow C_{S}^{2} a_{I} \\
& C_{I} \rightarrow C_{S}^{2} C_{I} \\
& C_{I I} \rightarrow C_{I I} / C_{S}^{2}
\end{aligned}
$$

and then take the limit $C_{S}^{2} \rightarrow 0$. The corresponding coupling coefficients then becomes

$$
\begin{aligned}
C_{I} & =-\frac{i}{2} \frac{n_{0} \omega_{I}}{k_{x I}} \omega_{g} \\
C_{I I} & =-\frac{i}{2} \frac{k_{x I} \omega_{I I}}{n_{0} \omega_{I}^{2}} \omega_{g}
\end{aligned}
$$

Another special case of particular interest is the limit of parallel propagation (but with arbitrary ratio $C_{S}^{2} / C_{A}^{2}$ ), in which case the magnetosonic dispersion relation coincides with that of the shear Alfvén wave, and the only distinction between the modes is the polarization, which differ by 90 degrees. Again the general coupling coefficient (31) seem to diverge, since from the magnetosonic dispersion relation $\omega_{I}^{2}-C_{A}^{2} k_{I}^{2} \sim k_{x I}^{2}$ when $k_{x I}^{2} \rightarrow 0$. However, by using another renormalization

$$
\begin{aligned}
& a_{I} \rightarrow a_{I} / \chi \\
& C_{I} \rightarrow C_{I} / \chi \\
& C_{I I} \rightarrow \chi C_{I I}
\end{aligned}
$$

and taking the limit $k_{I x}, k_{I I x} \rightarrow 0$, we obtain

$$
C_{I}=C_{I I}=-\frac{i}{2} \omega_{g}
$$

Since dissipation of the waves have not been included in our model, the instability threshold value of the gravitational amplitude found from (29) and (30) is so far zero. However, since only weak dissipation is of interest we can take such effects into account by simply substituting $\partial_{t} a_{\alpha} \rightarrow\left(\partial_{t}+\gamma_{\alpha}\right) a_{\alpha}$ in the coupled mode equations [25], where $\gamma_{\alpha}$ is the linear damping rate of the mode $\alpha$. The most common damping mechanism of MHD waves is that due to finite resistivity. Calculating the linear damping by replacing (12) with $\mathbf{E}+\mathbf{v} \times \mathbf{B}=\eta \mathbf{j}$, where $\eta$ is the resistivity, we find $\gamma_{\alpha}=\eta k_{\alpha}^{2} / \mu_{0}$. Next we introduce the (weakly time dependent) normal mode amplitudes, $A_{\alpha}$, defined by $a_{\alpha}=A_{\alpha} e^{-i \omega_{\alpha} t}$, where $\alpha=I, I I$. Substituting these expressions into (29) and (30) taking the damping into account, we find the general form for the condition of parametric growth of waves $\left|\widetilde{h}_{\times}\right|>$ $h_{\mathrm{thr}} \equiv\left(\gamma_{I} \gamma_{I I} / C_{I} C_{I I}^{*}\right)^{1 / 2}[22]$, where $\widetilde{h}_{\times}$is the amplitude of the gravitational wave and $h_{\mathrm{thr}}$ is the threshold value for parametric excitation. In the limit of parallel propagation we find from (35) that the threshold value $h_{\mathrm{thr}}$ reduces to

$$
\left|\widetilde{h}_{\times}\right|>h_{\mathrm{thr}} \approx \frac{4 \gamma_{I}, I I}{\omega_{g}}=\frac{\eta \omega_{g}}{\mu_{0} C_{A}^{2}}
$$

Furthermore, if the gravitational amplitude is well above threshold $\left(\left|\widetilde{h}_{\times}\right| \gg h_{\mathrm{thr}}\right)$ the general expression for the parametric growth rate $\Gamma$ from (29) and (30) is $\Gamma \approx$ $\sqrt{C_{I} C_{I I}^{*}}\left|\widetilde{h}_{\times}\right|[22]$, and the result for the special case of parallel propagation is

$$
\Gamma \approx \frac{1}{2} \omega_{g}\left|\widetilde{h}_{\times}\right|
$$

It should be pointed out that in addition to the wave interactions considered above, we have found zero coupling coefficients for a number of cases. To be specific: For the same polarization of the gravitational pump wave 
$\left(h_{+}=0\right)$, and propagation parallel to the external magnetic field, the following combinations of MHD-waves cannot be excited in the resonant three wave approximation, since the coupling coefficient then becomes zero: 1) Two ion-acoustic (or slow magnetosonic) modes. 2) One ion-acoustic and one Alfvén wave. 3) Two Alfvén waves with the same linear polarization.

Note that for the case of non-zero coupling considered above, the Alfvén waves have perpendicular polarizations in the parallel limit. The coupling between the differently polarized modes then results from the quadratic nonlinear terms proportional to $\dot{h}_{\times} v_{y} \hat{\mathbf{x}}$ and $\dot{h}_{\times} v_{x} \hat{\mathbf{y}}$ in eq. (11), and from similar cross terms in (16) that couple the two different polarizations through $h_{\times}$. The dependence of the results on the various polarizations for parallel propagation can be physically understood as follows: For the MHD-waves to gain energy from the gravitational wave, the MHD-waves must be able to reduce the gravitational wave amplitude. Including the source term in the wave equation for the gravitational wave, we immediately see that it is only the component $T_{x y}$ of the energy momentum tensor that may affect the gravitational wave with $h_{\times}$-polarization. However, for the three "null-cases" listed above, it is trivial to see that $T_{x y}$ vanishes (within the quadratic MHD approximation), and thus the gravitational wave is unaffected by the presence of such waves. From energy conservation [26] it is thus clear that the corresponding coupling must disappear. For perpendicular polarization of the Alfvén waves, on the other hand, $T_{x y}=m n_{0} v_{x} v_{y}-B_{x} B_{y} / \mu_{0} \neq 0$, and thus the gravitational amplitude is affected by such a combination of waves and in accordance with this we have found the coupling to be non-zero.

\section{SUMMARY AND DISCUSSION}

We have considered parametric excitation of Alfvén waves by gravitational radiation propagating parallel to the external magnetic field. As a starting point, standard ideal MHD equations (i.e. without special relativistic effects) incorporating the curvature of space time have been derived. It should be pointed out that the system of equations (5) - (8) in principle can be used in situations where we have strong deviation from Minkowski space time, although the condition of non-relativistic fluid velocities then limits the applicability. Focusing on the case where the metric is that of a small amplitude monochromatic gravitational wave superimposed on flat space-time, the growth rate for nonlinearly coupled shear Alfvén and fast magnetosonic waves have been found.

In our calculations we have considered a monochromatic gravitational pump wave, which could be produced by binary systems. As seen from (37) (or more generally from (31) and (32) which applies for arbitrary directions of propagations [27]), the growth rate is roughly of the order $\Gamma \sim h_{\times} \omega_{g}$. Thus the plasma parameters $n_{0}, B_{0}$ and $T$ do not significantly influence the growth rate, at least not as long as the assumptions of the derivation is fulfilled. It is not hard to find a plasma fulfilling these assumptions, e.g. choosing $n_{0} \sim 10^{14} \mathrm{~m}^{-3}[28]$ and considering waves with frequency $\omega \lesssim 10^{4} \mathrm{rad} \mathrm{s}^{-1}, B_{0}$ may attain any value roughly in the interval $10^{-5}-1 \mathrm{~T}$ for the estimate $\Gamma \sim h_{\times} \omega_{g}$ to apply, where the limits of the interval comes from the conditions $\omega \ll \omega_{c}$ and $C_{A}^{2} \ll c^{2}$ respectively. Furthermore, the condition $C_{S}^{2} \ll c^{2}$ still allows for comparatively high plasma temperatures, since it is obviously fulfilled provided the thermal velocities of the particles are much smaller than the speed of light. As for the source of gravitational radiation we follow Ref. 12 and consider a binary system of two equal masses $m=3 M_{\odot}$ separated by a distance of six Schwarzschild radii $r=12 G m / c^{2}$ emitting gravitational radiation with frequency of the order $\omega_{g} \approx 10^{4} \mathrm{rad} / \mathrm{s}$. By considering two point masses separated by a fixed distance $r$ the amplitude $\tilde{h}_{\times}$at a distance $R$ from the system is estimated to give $\tilde{h}_{\times} \sim G m r^{2} \omega_{g}^{2} /\left(2 c^{4} R\right)$. Also in the case of Ref.12, which considered parametric excitation of high-frequency plasma waves, the growth rate fulfilled $\Gamma \sim h_{\times} \omega_{g}$, implying the growth rate $\Gamma \sim 10^{-2} \mathrm{~s}^{-1}$ at a distance of $1 / 60$ au from the source, where a process at a closer distance was ruled out by the frequency matching conditions combined with the linear dispersion relations. In our case the linear dispersion relations and matching conditions allow a parametric process closer to the source, and thereby opens up the possibility for a higher growth rate, although too close to the source the background plasma may be too inhomogeneous and too far from steady state for our calculations to be applicable.

Furthermore, excitation of MHD waves may take place in a dense plasma, and therefore processes such as supernovas are of interest, where gravitational wave absorption may take place inside the exploding star. In a discussion of possible mechanisms of absorbing gravitational wave energy in supernovas Ref. 14 has written "Since the effect of acceleration by gravitational waves is independent of mass of the charge, both the ions and the electron respond in an identical manner, which is not the case for electromagnetic waves. This means that waves such as Alfvén waves which describe oscillation of charge neutral plasmas are ideal. The coupling, however, is weak." At the present stage of understanding it is too early to deduce whether significant gravitational wave absorption by MHD waves may occur. Calculations taking into account the effects of a broad band gravitational spectrum, plasma inhomogeneities, etc., must first be performed. In particular inhomogeneity scale lengths with a scale length significantly shorter than the wavelength of the gravitational mode - such as at the plasma boundary of the supernova - may lead to excitation of MHD surface waves with a significantly enhanced growth rate as compared to the present homogeneous plasma coupling 
mechanism. This is in analogy with parametric excitation scenarios for high frequency plasma surface waves [29], where the surface waves may have a considerably higher growth rate than the corresponding bulk waves, provided the inhomogeneity scale length is considerably shorter than the wave length of the pump wave. Such a problem, however, is a project for future work.

An interesting question from a theoretical point of view is whether the coupling coefficients $C_{I}$ and $C_{I I}$ of eqs. (31) and (32) satisfy the Manley-Rowe relations [22]. Generally such relations follow from an underlaying Hamiltonian structure of the governing equations, and assures that each of the decay products takes energy from the pump wave in direct proportion to their respective frequencies. This means that the parametric process can be interpreted quantum mechanically - i.e. we can think of a three wave process as the decay of a pump wave quanta with energy $\hbar \omega_{g}$ into wave quantas with energy $\hbar \omega_{I}$ and $\hbar \omega_{I I}$ respectively. An interesting consequence is that generally a lot of three wave decay processes are forbidden from the start by the Manley-Rowe relations (for example the decay of a plasmon into two photons), since they imply that we only get a positive growth rate when the pump wave has the highest frequency, in consistence with the quantum picture [30]. It should be pointed out that all well established basic systems of equations in plasma physics such as the ideal MHD-equations, the Vlasov-Maxwell equations and the standard multi-fluid equations, all possess the underlaying Hamiltonian structure that leads to fulfillment of the Manley-Rowe relations for three-wave interaction processes [31]. However, since the theory of gravitation differs in important respects from other fundamental theories of physics, it is an open question whether the Manley-Rowe relations applies also when one of the interacting modes is a gravitational wave. To investigate this issue we consider the energy increase rate of mode I. Multiplying Eq. (29) with $v_{x I}^{*} m k_{I}^{2} C_{s}^{2}\left(\omega_{I}-k_{z I}^{2} C_{A}^{2}\right) / \omega_{I} k_{x I}$ and using the eigenvectors (25) and (26) we find

$$
\frac{\partial W_{I}}{\partial t}=\omega_{I} V
$$

where $V=2 \operatorname{Im}\left[m n_{0} \omega_{g} h_{\times} v_{x I}^{*} v_{y I I}^{*} / \omega_{I}\right]$ and the magnetosonic wave energy density is $W_{I}=m n_{0} k_{I}^{2}\left(\omega_{I}-\right.$ $\left.k_{z I}^{2} C_{A}^{2}\right)\left(\partial D_{m} / \partial \omega_{I}\right)\left|v_{x I}\right|^{2} / 2 k_{x I}^{2} \omega_{I}$ (cf. Eq. (20) in Ref 32). Similarly, multiplying Eq. (30) with $m n_{0} v_{y I I}^{*}$, and applying (25) and (26) we obtain

$$
\frac{\partial W_{I I}}{\partial t}=\omega_{I I} V
$$

where $W_{I I}=m n_{0}\left|v_{y I I}\right|^{2}$ (cf. Eq. (13) in Ref 32). Up to now, we have treated the gravitational wave as a pump wave, i.e. we have only considered the initial stage of an instability where the energy density of the daughter waves are small enough for the influence on the gravitational wave to be neglected. For a practical purpose this regime may apply until nonlinear saturation mechanisms (outside our calculation scheme) sets in for the growing MHD-waves, unless the plasma is extremely dense. From a theoretical point of view, however, it is of interest to consider the decrease in the gravitational wave amplitude due to the growth of the MHD perturbations. Including the energy momentum source term in the wave equation for the gravitational wave, i.e.using $\square h_{\mu \nu}=-2 \kappa T_{\mu \nu} \equiv$ $-16 \pi G T_{\mu \nu} / c^{4}$, keeping only the resonantly varying part of $T_{\mu \nu}$ (i.e. the part proportional to $\exp \left[\mathrm{i}\left(k_{g} z-\omega_{g} t\right)\right]$ ) and letting $h_{12}=\widetilde{h}_{\times}(t) \exp \left[\mathrm{i}\left(k_{g} z-\omega_{g} t\right)\right]+$ c.c., we immediately obtain

$$
\mathrm{i} \omega_{g} \frac{\partial \widetilde{h}_{\times}}{\partial t}=\kappa\left(T_{12}\right)_{\mathbf{k}_{g}}=\kappa\left[m n_{0} v_{x I} v_{y I I}-\frac{B_{x I} B_{y I I}}{\mu_{0}}\right]
$$

which, after using the eigenvectors (25) and (26) and multiplying with $\omega_{g} \widetilde{h}_{\times}^{*}$ can be written

$$
\frac{\partial W_{g}}{\partial t}=-\omega_{g} V
$$

where $W_{g}=\omega_{g}^{2}\left|\widetilde{h}_{\times}\right|^{2} / 2 \kappa$ is the energy density of the gravitational wave [33]. Since the same factor $V$ appears in Eqs (38), (39) and (41), the Manley-Rowe relations are indeed fulfilled (i.e. each mode changes energy in direct proportion to its frequency), and furthermore we see that the total wave energy $W=W_{I}+W_{I I}+W_{g}$ is conserved, which follows from (38), (39) and (41) together with the frequency matching condition (27).

[1] Yu. G. Ignat'ev, Phys. Lett. A 230, 171 (1997).

[2] V. I. Denisov, Zh. Eksp. Teor. Fiz. 74, 401 (1978). [Sov. Phys. - JETP 42, 209 (1978)].

[3] M. Demiański, Relativistic Astrophysics, pp 256-257 (Pergamon Press, 1985).

[4] M. Marklund, G. Brodin and P. K. S. Dunsby, Astrophys. J. 536, 875 (2000).

[5] U. N. Gerlach, Phys. Rev. Lett. 32, 1023 (1974).

[6] M. Marklund, P. K. S. Dunsby and G. Brodin, To appear in Phys. Rev. D in the issue of 15 october, 2000 .

[7] G. A. Lupanov, Zh. Eksp. Teor. Fiz. 52, 118 (1967).[Sov. Phys. - JETP 25, 76 (1967)].

[8] V. B. Braginskiǔ, L. P. Grishchuk, A. G. Doroshkevich, Ya. B. Zel'dovich, I. D. Novikov and M. V. Sazhin, Zh. Eksp. Teor. Fiz. 65, 1729, (1973).[ Sov. Phys. - JETP 38, 865 (1974)].

[9] L. P. Grishchuk and M. V. Sazhin, Zh. Eksp. Teor. Fiz. 68, 1569 (1975).[Sov. Phys. - JETP 41, 787 (1976)].

[10] A. B. Balakin and Yu. G. Ignat'ev, Phys. Lett. A 96, 10 (1983).

[11] Yu. G. Ignat'ev, Zh. Eksp. Teor. Fiz. 81, 3 (1981) [Sov. Phys. JETP 54, 1 (1981)]. 
[12] G. Brodin and M. Marklund, Phys. Rev. Lett. 82, 3012 (1999).

[13] Mendonça, J. T., Shukla, P. K. and Bingham, R., Phys. Lett. A 250, 144 (1998).

[14] R. Bingham et al. Phys. Scripta, T75, 61 (1998).

[15] F. I. Cooperstock, Ann. Phys. 47, 173 (1968).

[16] Ya. B. Zeldovich, Zh. Eksp. Teor. Fiz. 65, 1311 (1973).[ Sov. Phys. - JETP 38, 652 (1974)]

[17] L. P. Grishchuk and A. G. Polnarev, Gravitational waves and their interaction with matter and fields, in General Relativity and Gravitation (Vol 2), ed. A. Held (Plenum Press, 1980).

[18] A. M. Anile, J. K. Hunter and B. Truong, J. Math. Phys. 40, 4474 (1999).

[19] A. Greco, and L. Seta, J. Class. Ouantum Grav. 15, 3655 (1998).

[20] We could also have started from previous general relativistic MHD-equations - see e.g. A. M. Anile, Relativistic fluids and magneto-fluids (Cambridge University Press 1989) - but the present derivation has a value as a guide for future generalizations to the case of general relativistic two-fluid equations.

[21] Naturally the condition $\partial / \partial t \ll \omega_{c}$ cannot hold in arbitrary reference frames. We assume it to be true in the rest frame of the fluid, but due the condition of nonrelativistic fluid velocities it also holds in all frames of relevance.

[22] J. Weiland and H. Wilhelmsson, Coherent Nonlinear Interaction of Waves in Plasmas, (Pergamon press 1977).

[23] This is an additional restriction as compared to the general case, since the choice of gravitational polarization (i.e. letting $h_{+}=0$ ) makes the $x$ - and $y$-axis non equivalent.

[24] In order to check the algebra we have verified that identical coupling coefficents $C_{I}$ and $C_{I I}$ follows from a tetrad formalism.

[25] We assume that terms that are both nonlinear and proportional to the small dissipation parameter is negligible.

[26] Generally it is not entirely unproblematic to make energy conservation arguments in general relativity. However, as will be clear from the last section, energy conservation using the standard expression for the gravitational wave energy can be applied to our case.

[27] Using $\Gamma=\sqrt{C_{I} C_{I I}^{*}} h_{\times} \omega_{g}$ and estimating $C_{I}$ and $C_{I I}$ as given by (31) and (32), applying the dispersion relations and the frequency matching conditions, we get $\Gamma \sim h_{\times} \omega_{g}$ also for non-parallel propagation and independently of the ratio $C_{S}^{2} / C_{A}^{2}$.

[28] The possible particle densities in the example of considerations have a very wide range. For example $n_{0} \sim$ $10^{4} \mathrm{~m}^{-3}$ for low-density unperturbed interstellar matter. The value $n_{0} \sim 10^{14} \mathrm{~m}^{-3}$ chosen by us somewhat arbitrarily, is of the some order of magnitude as the density in the solar corona. Much larger number densities, as found in accretion discs, are also possible.

[29] G. Brodin and J. Lundberg, J. Plasma Phys., 46, 299 (1991).

[30] Note that the even in standard plasma problems, the quantum picture should not be taken too literally, since the process is indeed nonlinear, and the interaction of single wave quantas thus is negligible.

[31] J. Larsson, Phys. Scripta, 58, 97 (1998).

[32] G. Brodin and L. Stenflo, J. Plasma Phys.,39, 277 (1988).

[33] L.D. Landau and E.M. Lifshitz, The Classical Theory of Fields (Pergamon Press, Oxford 1975). 